DOI: 10.14451/1.167.41

\title{
ЧИСЛЕННОЕ ИССЛЕДОВАНИЕ ПРОБЛЕМЫ СОГЛАСОВАННОГО УПРАВЛЕНИЯ ПРОЕКТАМИ ПО ОСВОЕНИЮ НОВОЙ ПРОДУКЦИИ
}

\author{
(c) 2018 Павлов Олег Валерьевич \\ кандидат технических наук, доцент \\ заместитель директора института экономики и управления \\ Самарский национальный исследовательский университет имени академика С.П. Королёва \\ 443086, г. Самара, ул. Московское шоссе, д. 34 \\ E-mail:pavlov@ssau.ru
}

\begin{abstract}
В работе рассматривается задача управления проектом по освоению новой продукции на промышленном предприятии. В процессе освоения новой продукции проявляется эффект обучения, который заключается в том, что затраты времени рабочих на выполнение многократно повторяющихся задач снижаются, что приводит к динамическому изменению экономических показателей производства. Проект освоения новой продукции рассматривается как управляемая иерархическая динамическая система, состоящая из руководства проекта и коллективов исполнителей. Для решения задачи применен принцип компенсации затрат, который разделяет исходную задачу на задачи согласованного стимулирования и согласованного планирования. Задача согласованного планирования математически формализуется как задача оптимального управления процессом в дискретном времени. Получены численные решения задач согласованного планирования для различных моделей кривых обучения с помощью метода динамического программирования Беллмана. На основе численного исследования сформулированы рекомендации по выбору функции стимулирования и плановых траекторий объемов производства
\end{abstract}

Ключевые слова: модели кривых обучения, согласованные задачи стимулирования, согласованные задачи планирования.

\section{Введение}

В проектах по освоению новой продукции на промышленных предприятиях проявляется эффект кривой обучения, который заключается в том, что затраты времени работников (трудоемкость) на выполнение многократно повторяющихся производственных задач снижаются.

Проект освоения новой продукции рассматривается как управляемая иерархическая динамическая система, состоящая из руководства проекта (центра) и исполнителей (агентов). Динамика управляемой динамической производственной системы зависит только от действий агента, а центр выбором функции материального стимулирования оказывает воздействие на целевую функцию агента. Состояние иерархической динамической системы в каждый период времени зависит от её состояния и действий участников в предыдущий период. Производственная деятельность в проекте по освоению нового производства характеризуется несовпадающими интересами центра и агентов, что приводит к снижению экономической эффективности. Разрешить эти противоречия воз- можно с помощью согласованных механизмов управления, которые побуждают агентов к выбору действий выгодных центру.

Динамические модели взаимодействия неравноправных игроков рассматриваются в теории активных систем [1], информационной теории иерархических систем [2], динамических игр [3]. Прикладные модели теории динамических игр в области экономики и менеджмента приводятся в работах [4-5].

В рассматриваемой динамической игровой модели присутствуют динамика принятия решений и динамика управляемой системы. Неравноправие участников фиксируется порядком ходов, первый ход делает центр, выбирая свою стратегию: функцию стимулирования и сообщая ее агентам. Центр, зная целевые функции агентов, максимизирует свою целевую функцию с учетом оптимальных ответов агентов. Предполагается, что агенты не связаны друг с другом и выполняют действия независимо. Динамическая игровая модель рассматривается в дискретной форме, что отражает характер производственной деятельности. 
1. Постановка динамической задачи управления проектами по освоению новой продукции

Рассматривается двухуровневая динамическая производственная система, состоящая из центра и $n$ независимых агентов. Агенты производят комплектующие детали, из которых затем собирается готовое изделие. Трудовые затраты и материальное стимулирование агентов зависят только от их собственных действий. В работе применяется принцип декомпозиции игры [1], который позволяет рассматривать управление i-ым агентом независимо и не учитывать взаимодействие агентов между собой. Поэтому рассматривается задача управления только одним $i$-ым агентом. Центр не занимается производственной деятельностью, распределяет плановый объем работы $i$-му агенту и контролирует его выполнение.

Динамика производственного процесса изготовления детали $i$-ым агентом описывается дискретным уравнением:

$$
x_{t}=x_{t-1}+u_{t}, \quad t=1, T
$$

где $x_{t}-$ кумулятивный объём производства детали за $t$-й временной период, $t$ - номер временного периода, $u_{t}$ - объём производства детали в периоде $t, T$ - количество рассматриваемых периодов (горизонт планирования).

В начальный период известно количество произведенных деталей:

$$
x_{0}=X_{0}
$$

В конечный период кумулятивный объём готовых деталей должен быть равен заданному центром:

$$
x_{T}=X_{0}+R
$$

где $R$ - заданное количество деталей центром агенту.

На объём производства детали в каждом периоде $t$ наложены следующие ограничения:

$$
0 \leq u_{t} \leq X_{0}+R-X_{t-1}, t=1, T
$$

В качестве целевой функции центра рассматривается максимизация суммарного дисконтированного дохода центра, разницы между доходом от произведенного агентом детали и затратами на материальное стимулирование агента:

$$
J_{p}=\sum_{t=1}^{T} \frac{1}{\left(1+r_{p}\right)^{t}}\left[p u_{t}-\sigma\left(x_{t}\right)\right] \rightarrow \max ,
$$

где $p$ - цена детали, $\sigma\left(x_{t}\right)-$ функция стимулирования центра, $r_{p}-$ ставка дисконтирования центра.

Под функцией стимулирования понимается правило выплаты материального вознаграждения агенту за выполненный объем работы. Центр воздействует на производственный процесс через механизм материального стимулирования $\sigma\left(x_{t}\right)$, экономически заинтересовывая агентов выполнять плановые объемы производства.

Целевой функцией агента является максимизация суммарного дисконтированного дохода:

$$
J_{a}=\sum_{t=1}^{T} \frac{1}{\left(1+r_{a}\right)^{t}}\left[\sigma\left(x_{t}\right)-C_{t}\left(u_{t}, x_{t-1}\right)\right] \rightarrow \max ,
$$

где $r_{a}$ - ставка дисконтирования агента, $C_{t}\left(u_{t}, x_{t-1}\right)$ - трудовые затраты агента.

Доход агента - это разница между материальным стимулированием и его трудовыми затратами, выраженными в денежной форме.

Трудовые затраты агента в периоде $t$ определяются как произведение трудоемкости $c_{t}$, объёма производства в этом периоде $u_{t}$ и стоимости одного норма часа s:

$$
C_{t}\left(u_{t}, x_{t-1}\right)=s C_{t} u_{t}
$$

Динамика изменения трудоемкости от кумулятивного объёма производства описывается различными моделями кривой обучения. Наиболее типичными моделями являются степенная, экспоненциальная и логистическая, приведенные в научной литературе [6]-[9].

Степенная модель трудоемкости:

$$
c_{t}=a x_{t-1}^{-b} \text {. }
$$

где $a-$ затраты на производство первого изделия, $b$ - индекс обучения.

Индекс обучения характеризует темп снижения трудоемкости агента при увеличении кумулятивного объёма производства.

Экспоненциальная модель трудоемкости:

$$
c_{t}=k+\beta e^{-\alpha x_{t-1}}
$$


где $\alpha-$ индекс обучения, $k, \beta-$ параметры экспоненциальной модели.

Логистическая модель трудоемкости:

$$
c_{t}=c_{\min }+\left(c_{\max }-c_{\min }\right)\left[\frac{1}{1+\beta e^{\alpha \mathrm{x}_{\mathrm{t}-1}}}\right]
$$

где $c_{\min }, c_{\max }-$ минимальные и максимальные значения трудоемкости, $\alpha-$ индекс обучения, $\beta$ - параметр логистической модели.

Задача управления центра заключается в выборе оптимальной системы стимулирования $\sigma\left(x_{t}\right)$, при которой агент выберет оптимальные объёмы производства деталей $u_{t}^{o p t}, t=1, n$ удовлетворяющие ограничению (4), которые осуществят перевод производственного процесса (1) из начального состояния (2) в конечное состояние (3) и максимизируют суммарный дисконтированный доход центра (5).

Так как у агента есть собственный экономический интерес, то реакцией агента на систему стимулирования центра является выбор оптимальных объёмов производства деталей $u_{t}^{\text {opt }}, t=1, n$ удовлетворяющих ограничению (4), которые осуществляют перевод производственного процесса (1) из начального состояния (2) в конечное состояние (3) и максимизируют суммарный дисконтированный доход агента (6).

2. Алгоритм решения динамической задачи управления проектами по освоению новой продукции

Для решения сформулированной задачи управления применяется принцип компенсации затрат [1]. В соответствии с принципом компенсации затрат для того, что бы побудить агента выбрать плановую траекторию центру достаточно компенсировать его затраты:

$$
\sigma\left(x_{t}\right)=C_{t}\left(u_{t}, x_{t-1}\right)
$$

Целевая функция центра примет вид:

$$
J_{p}=\sum_{t=1}^{T} \frac{1}{\left(1+r_{p}\right)^{t}}\left[p u_{t}-C_{t}\left(u_{t}, x_{t-1}\right)\right] \rightarrow \max .
$$

С учетом (7) целевая функция центра запишется:

$$
J_{p}=\sum_{t=1}^{T} \frac{1}{\left(1+r_{p}\right)^{t}}\left[p-s c_{t}\right] u_{t} \rightarrow \max
$$

Учитывая, что цена детали $p$ и стоимость нормо-часа $s$ постоянные величины, максимизацию суммарного дохода центра можно заменить минимизацией трудовых затрат агента.

$$
J_{p}=\sum_{t=1}^{T} \frac{1}{\left(1+r_{p}\right)^{t}} C_{t} u_{t} \rightarrow \min .
$$

Алгоритм решения состоит в разделении исходной задачи на задачу согласованного стимулирования и задачу согласованного планирования.

1. Задача согласованного динамического стимулирования.

Центр выбирает компенсаторную систему стимулирования, которая заключается в компенсации затрат агента в случае выбора оптимальной плановой траектории центра $x_{t}^{R}, t=1, T$ и отсутствия материальных выплат в противном случае:

$$
\sigma\left(x_{t}\right)=\left\{\begin{array}{l}
C_{t}\left(u_{t}, x_{t-1}\right), \text { если } x_{t}=x_{t}^{R}, \text { для } \forall t=1, T, \\
0, \quad \text { если } x_{t} \neq x_{t}^{R}, \text { для } \forall t=1, T .
\end{array}\right.
$$

В практической деятельности компенсаторная система материального стимулирования может быть реализована в виде штрафа за отклонение от плановой траектории:

$$
\sigma\left(x_{t}\right)=Z_{t}-Z_{t} \lambda a b s\left(1-\frac{x_{t}}{x_{t}^{R}}\right),
$$

где $Z_{t}$ - материальное вознаграждение агента, равное еготрудовым затратам: $Z_{t}=C_{t}\left(u_{t}, x_{t-1}\right)$

, $\lambda$ - параметр системы стимулирования.

2. Задача согласованного динамического планирования.

Оптимальная плановая траектория центра $x_{t}^{R}, t=1, T$ определяется из решения задачи оптимального управления:

$$
\begin{aligned}
& J_{p}=\sum_{t=1}^{T} \frac{1}{\left(1+r_{p}\right)^{t}} c_{t} u_{t} \rightarrow \min . \\
& x_{t}=x_{t-1}+u_{t}, \quad t=1, T,
\end{aligned}
$$

$$
x_{0}=X_{0}
$$

$$
x_{T}=X_{0}+R
$$

$$
0 \leq u_{t} \leq X_{0}+R-x_{t-1}, t=1, T
$$

При использовании компенсаторной системы стимулирования задача управления центра сведена к задаче оптимального управления. За- 
дача центра заключается в выборе оптимальных объёмов производства деталей $u_{t}^{o p t}, t=1, n$ удовлетворяющие ограничению (12), которые осуществят перевод производственного процесса (9) из начального состояния (10) в конечное состояние (11) и минимизируют суммарные дисконтированные затраты агента (8).

Для численного решения сформулированной задачи применялся метод динамического программирования Беллмана [10]-[11], реализованный на языке программирования pascal.

\section{2. Результаты решения согласованной} задачи планирования

Численное решение согласованной задачи планирования проведено на примере производства новых изделий предприятия АО «Салют». По данным предприятия построены регрессионные модели трудоемкости новых комплектующих деталей: «Кассета», «Балка» и «Отсек».

Трудоемкость производства комплектующего «Кассета» описывается степенной моделью:

$$
c_{t}=42,64 x_{t-1}^{-0,29} \text {. }
$$

Трудоемкость производства комплектующего «Балка» описывается экспоненциальной моделью:

$$
c_{t}=9,17+6,16 e^{-0,0169 x_{t-1}} .
$$

Трудоемкость производства комплектующего «Отсек» описывается логистической моделью:

$$
c_{t}=55,10+36,61\left[\frac{1}{1+0,017 e^{0,0561 x_{t-1}}}\right] .
$$

Для исследования использовались следующие данные: заданный объём производства изделий $R=240$ шт., количество временных периодов $T=12$ месяцев, производственный опыт подразделения до первого периода $x_{0}=1$ шт. Дискретный шаг изменения объемов производства при проведении численных расчетов выбирался 1 комплектующая деталь. Ставка дисконтирования центра при расчетах принималась равной нулю.

Численные решения задачи согласованного планирования для степенной, экспоненциальной и логистической модели кривой обучения представлены на рисунках 1-8. На рисунках представлены зависимости оптимальных кумулятивных объемов производства и оптимальных объемов производства от временных периодов для различных индексов обучения.

Анализируя рис. 1-4 приходим к выводу, что для степенной и экспоненциальной моделей кривых обучения оптимальной траекторией кумулятивного объема производства является выпуклая кривая. Оптимальной стратегией менеджмента предприятия является увеличение объёмов производства изделия от минимального значения в первом периоде до максимального в последнем периоде. С увеличением индекса обучения для степенной и экспоненциальной моделей оптимальная траектория кумулятивного объёма производства становится более выпуклой.

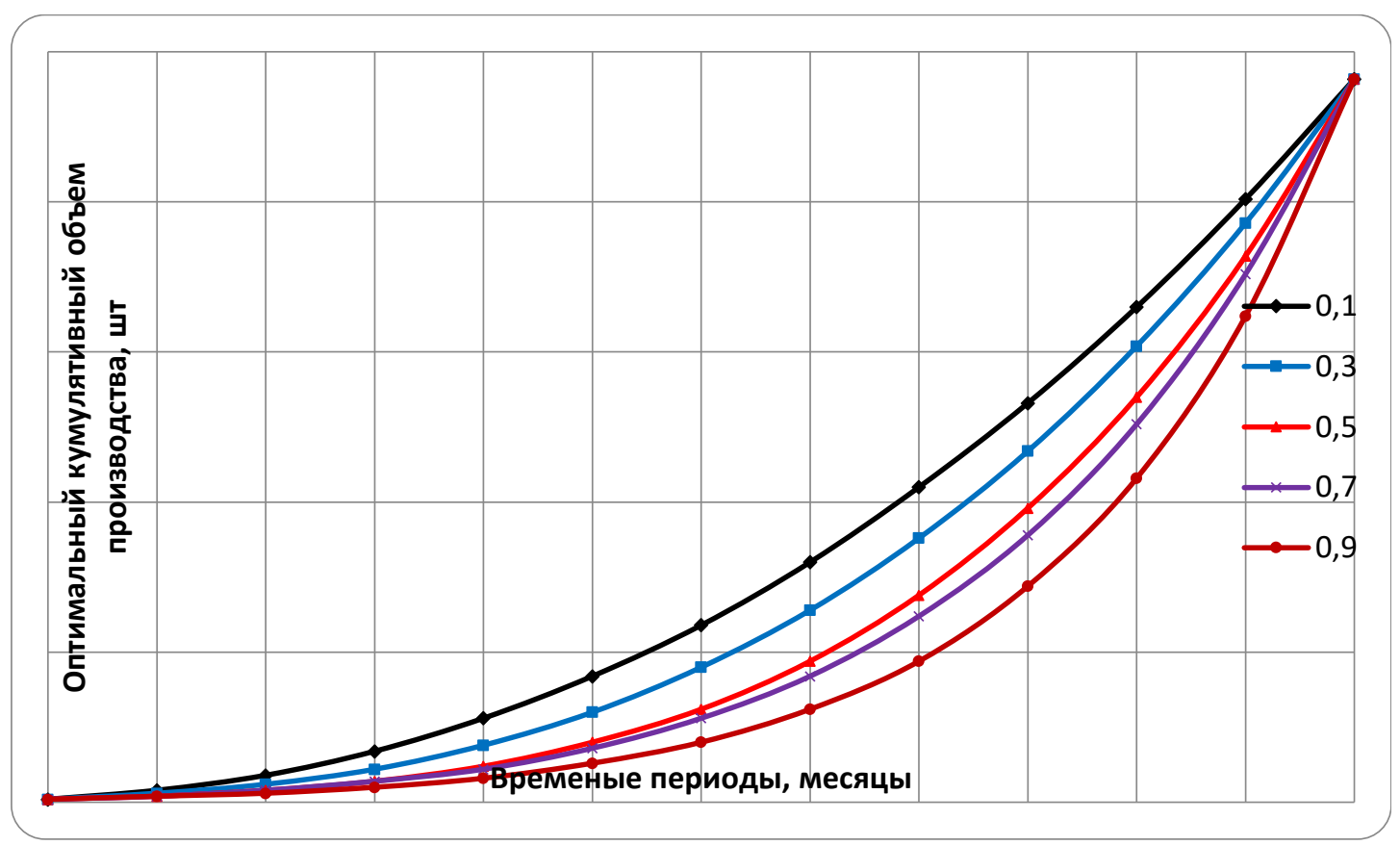

Puc. 1. Зависимость оптимального кумулятивного объёма производства от индекса обучения для степенной модели кривой обучения 


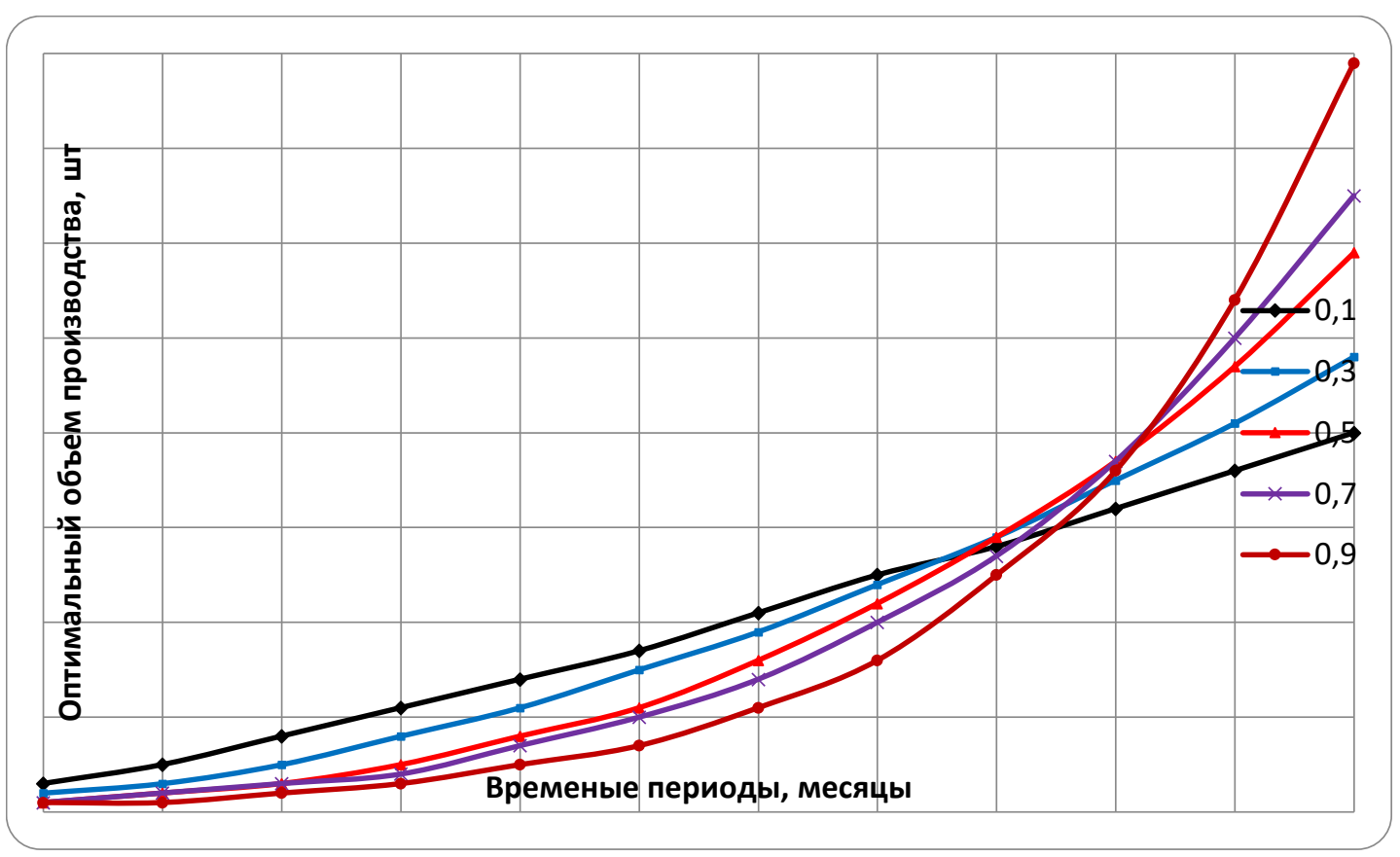

Puc. 2. Зависимость оптимальных объёмов производства от индекса обучения для степенной модели кривой обучения

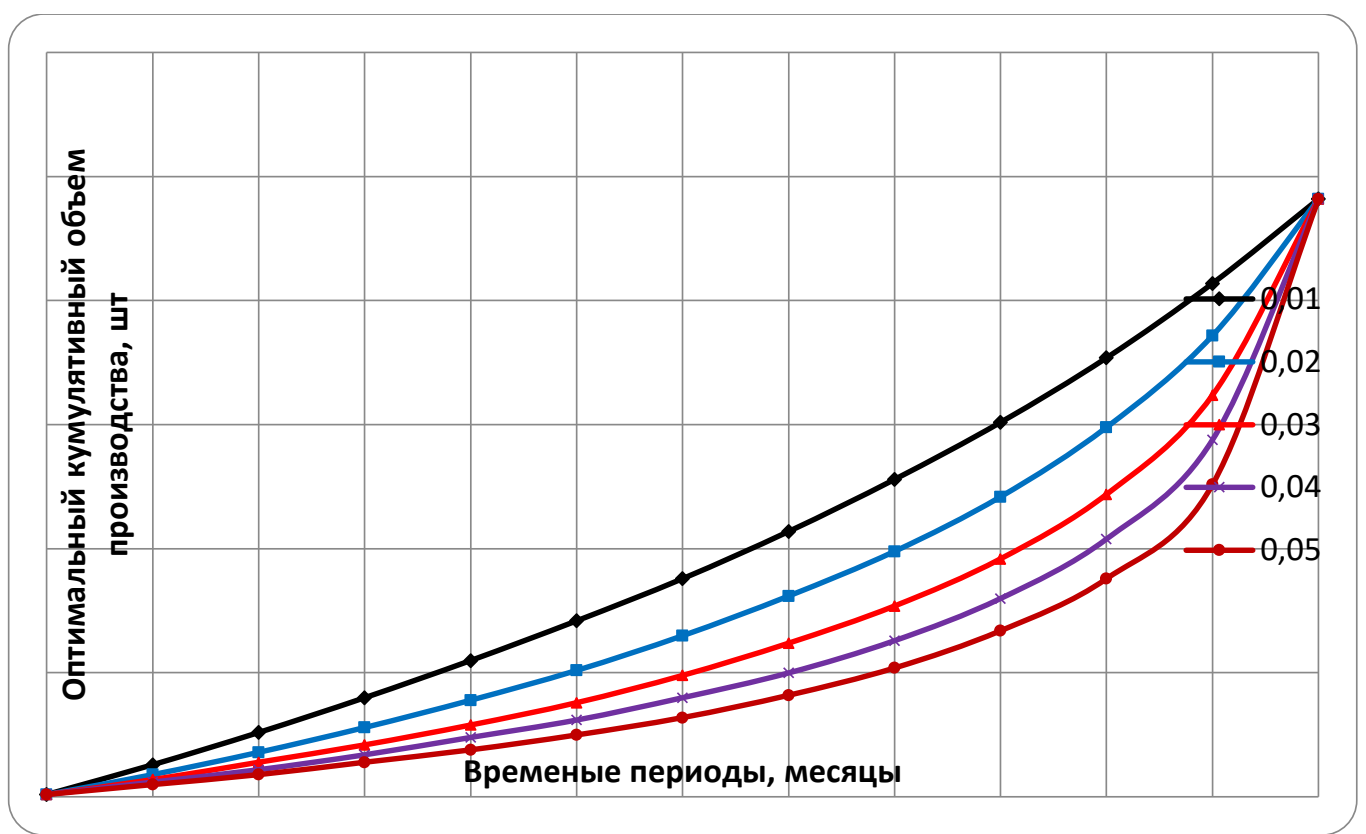

Puc. 3. Зависимость оптимального кумулятивного объёма производства от индекса обучения для экспоненциальной модели кривой обучения 


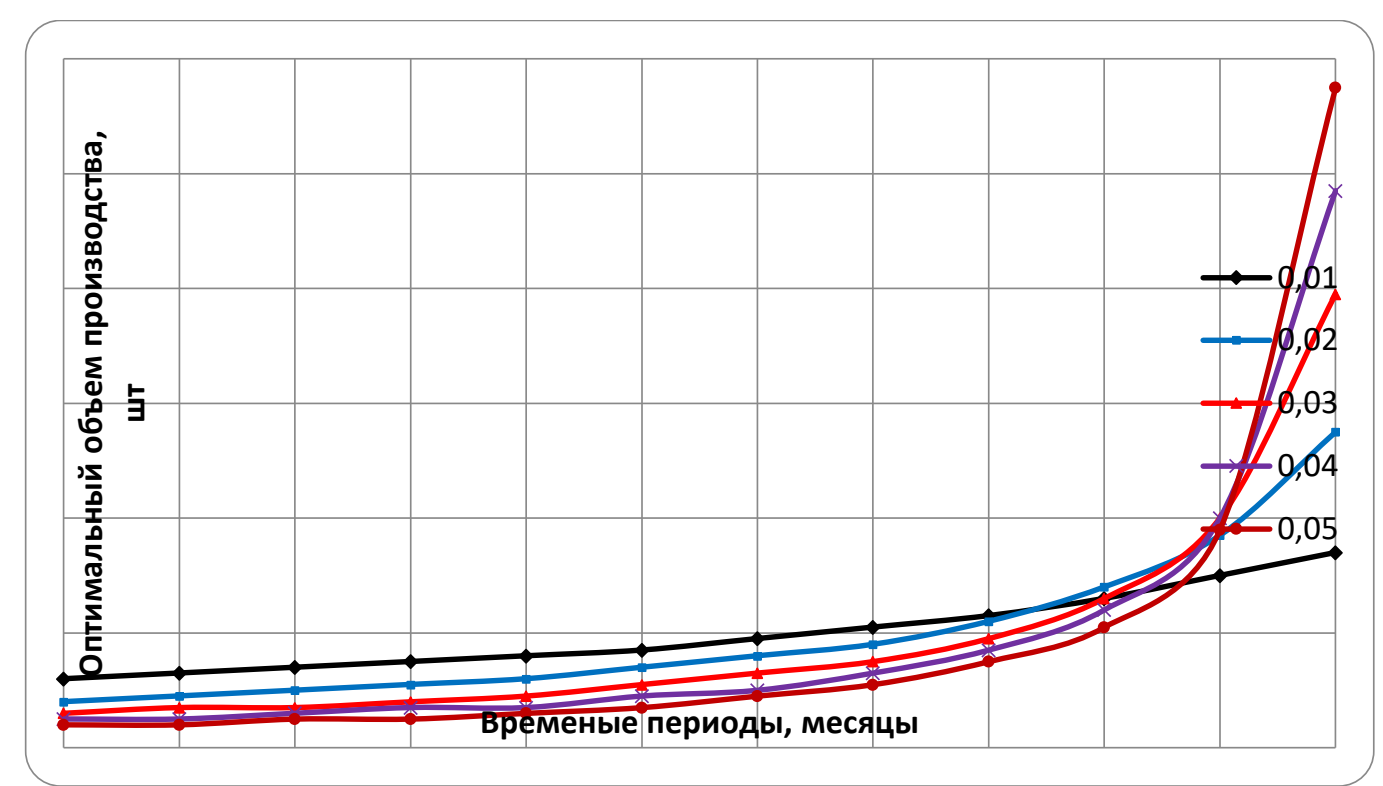

Puc. 4. Зависимость оптимальных объёмов производства от индекса обучения для экспоненциальной модели кривой обучения

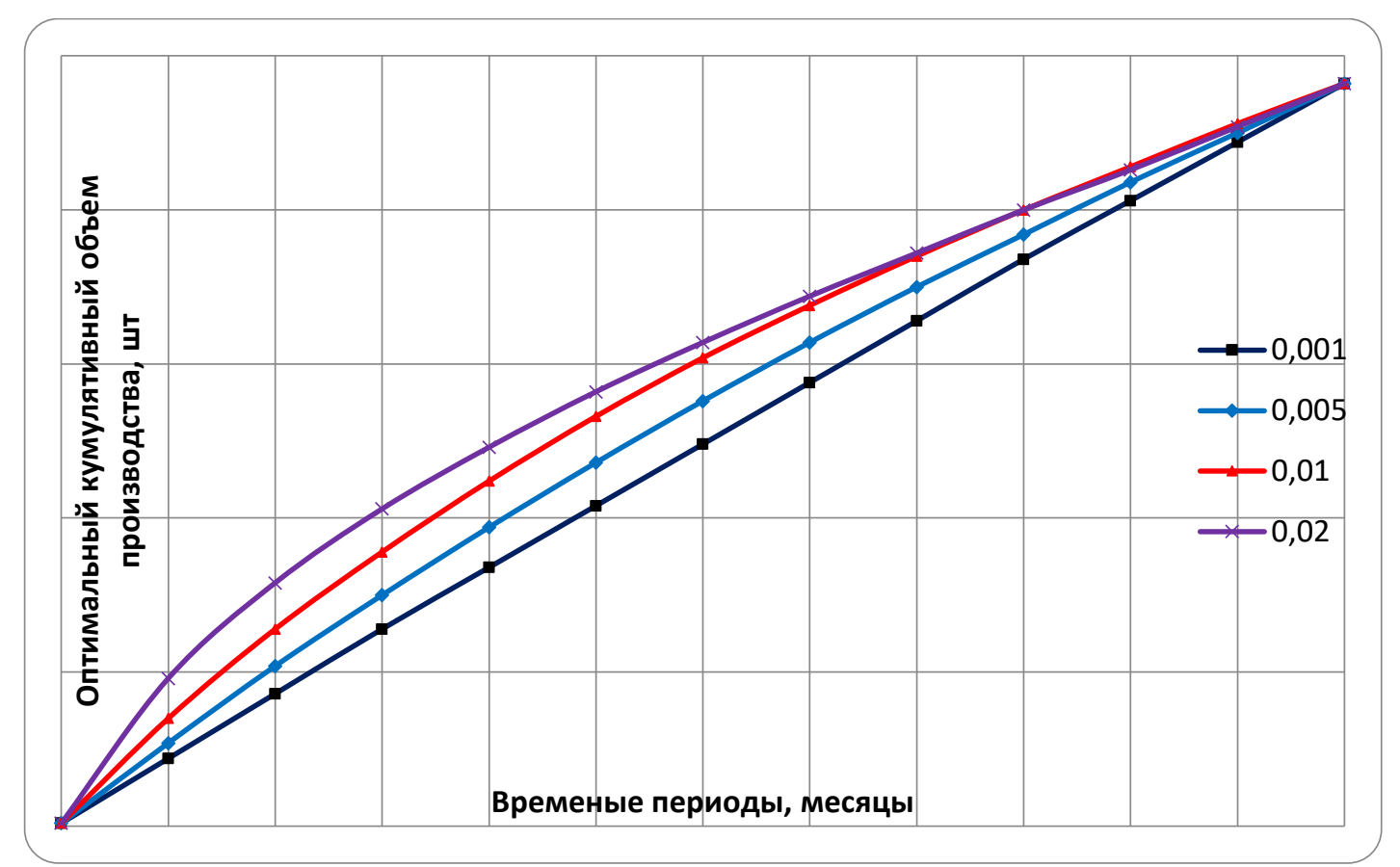

Puc. 5. Зависимость оптимального кумулятивного объёма производства от индекса обучения для логистической модели кривой обучения 


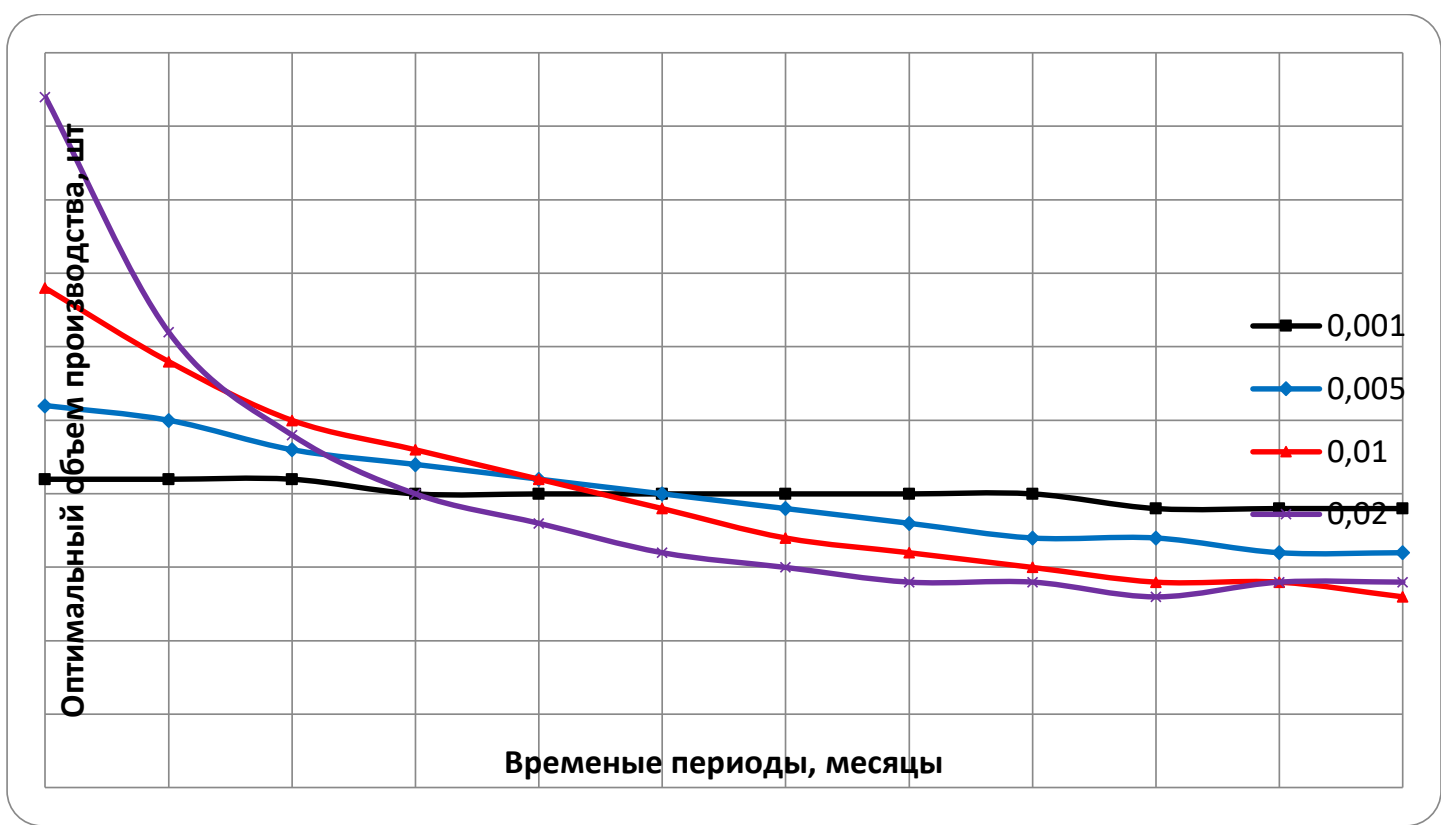

Puc. 6. Зависимость оптимальных объёмов производства от индекса обучения для логистической модели кривой обучения

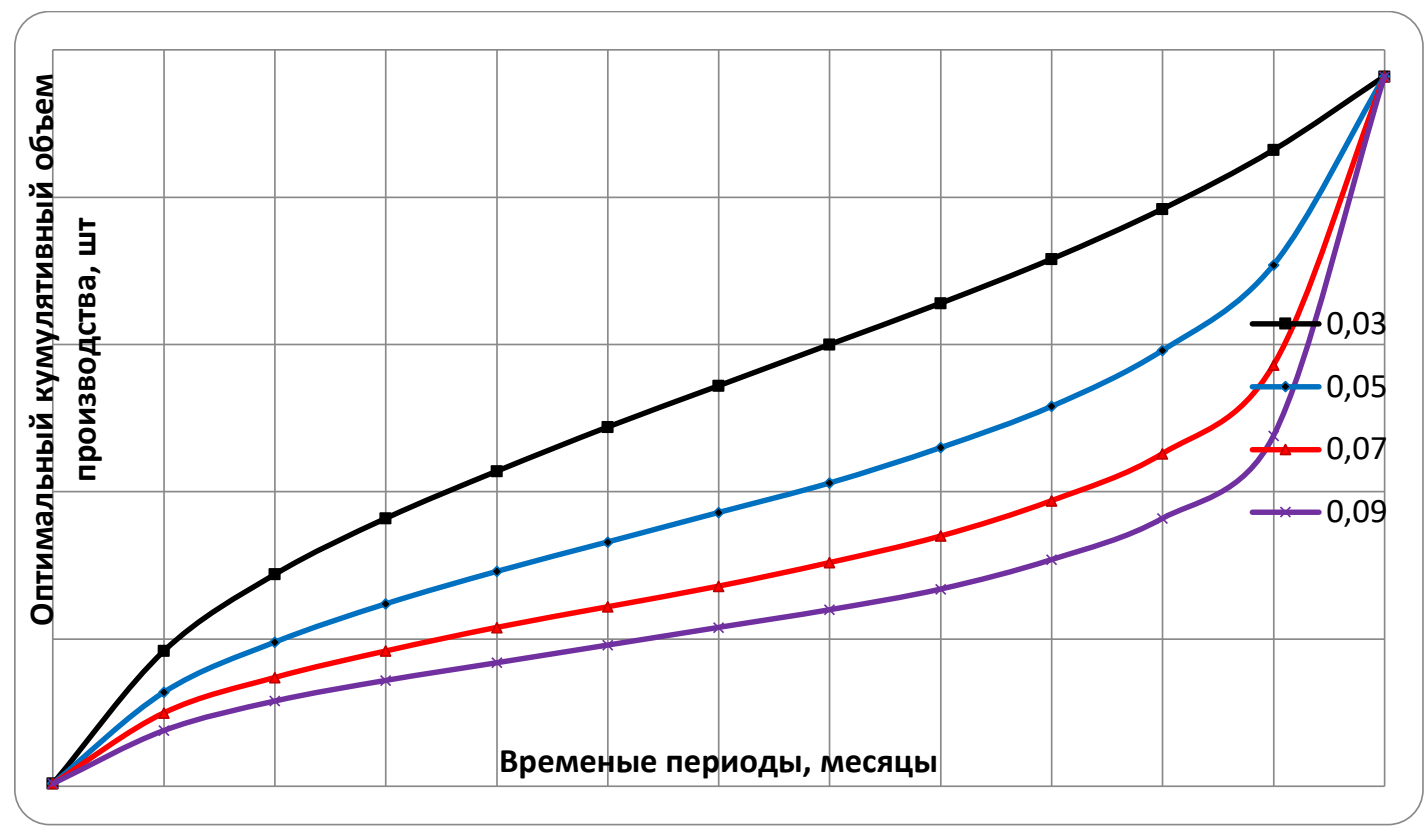

Puc. 7. Зависимость оптимального кумулятивного объёма производства от индекса обучения для логистической модели кривой обучения 


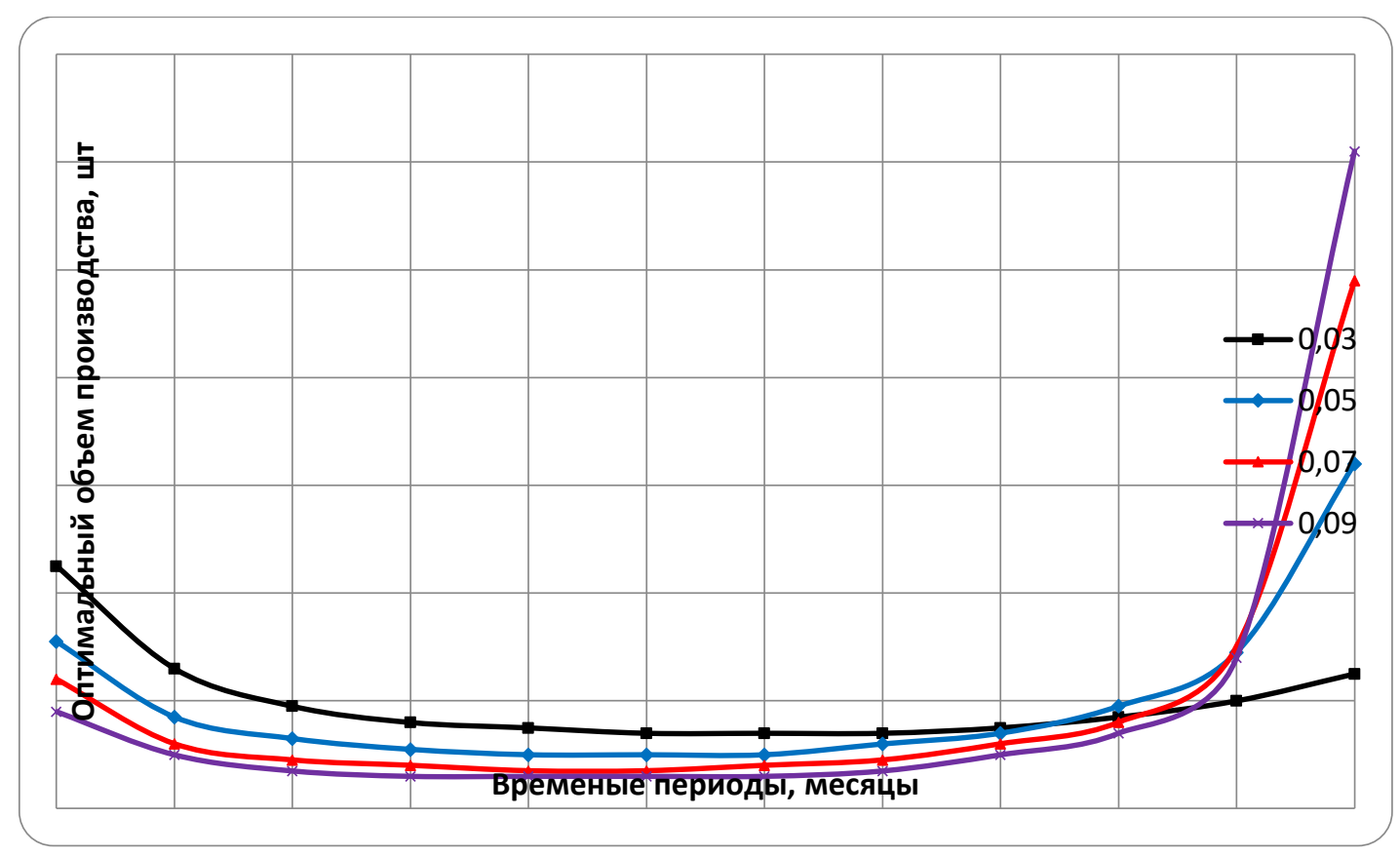

Рuс. 8. Зависимость оптимальных объёмов производства от индекса обучения для логистической модели кривой обучения

Анализируя рисунок 5 приходим к выводу, что для логистической модели кривой обучения с индексом обучения $\alpha<0,02$ оптимальной траекторией кумулятивного объема производства является вогнутая кривая. С увеличением индекса обучения оптимальная траектория кумулятивного объёма производства становится более вогнутой. Из анализа рис. 6 делаем вывод, что оптимальной стратегией менеджмента предприятия является уменьшение объемов производства от максимального значения в первом периоде до минимального в последнем периоде. При индексе обучения $\alpha=0,02$ оптимальная стратегия изменяется, уменьшение объёмов производства в последних периодах изменяется на увеличение.

Анализируя рисунок 7 приходим к выводу, что для логистической модели кривой обучения с индексом обучения $\alpha \geq 0,02$ оптимальной траекторией кумулятивного объема производства является логистическая кривая. Из анализа рис. 8 делаем вывод, что оптимальной стратегией менеджмента предприятия для логистической модели кривой обучения является сначала уменьшение объемов производства до минимального значения, а затем увеличение объемов производства до максимального значения. Вогнутому участку траектории кумулятивного объема производства соответствует уменьшение объемов производства, выпуклому участку траектории - увеличение объемов производства. Минимальный объем производства соответствует точке перегиба траектории кумулятивного объема. С увеличением индекса обучения оптимальные объемы производства значительно увеличиваются в последнем периоде и уменьшаются во всех остальных. Чем больше индекс обучения, тем больший рост объемов производства в последнем периоде.

\section{Заключение}

В работе сформулирована задача управления агентами в проектах по освоению новой продукции в дискретном времени.

Для решения задачи применен принцип компенсации затрат, который разделяет исходную задачу на задачи согласованного стимулирования и согласованного планирования. Задача согласованного планирования математически формализуется как задача оптимального управления процессом в дискретном времени. Показано, что задача управления центра сводится к выбору плановой траектории объемов производства с целью минимизации трудовых затрат агентов.

Получены численные решения задач согласованного планирования для различных моделей кривых обучения с помощью метода динамического программирования Беллмана. На основе численного исследования сформули- 
рованы рекомендации по выбору функции стимулирования и плановых траекторий объемов производства:

1. Оптимальной системой материального стимулирования является компенсаторная система. При использовании компенсаторной системы затраты менеджмента проекта на материальное стимулирование являются минимальными. В практической деятельности компенсаторная система может быть реализована в виде штрафов за отклонение от плановой траектории.

2. Для степенной и экспоненциальной моделей кривых обучения оптимальной стратегией менеджмента проекта является увеличение объёмов производства изделия от минимального значения в первом периоде до максимального в последнем периоде.

3. Для логистической модели кривой об- учения с маленьким индексом обучения оптимальной стратегией менеджмента проекта является уменьшение объемов производства от максимального значения в первом периоде до минимального в последнем периоде.

4. Для логистической модели кривой обучения с большим индексом обучения оптимальной стратегией менеджмента проекта является вначале уменьшение объемов производства до минимального значения, а затем увеличение объемов производства до максимального значения. Минимальный объем производства соответствует точке перегиба траектории кумулятивного объема производства.

*Исследование выполнено при финансовой поддержке РФФИ и Самарской области в рамках научного проекта № 17-46-630606.

\section{Библиографический список}

1. Новиков Д.А. Механизмы управления динамическими активными системами / Д.А. Новиков, И.М. Смирнов, Т.Е. Шохина. Москва. 2002. 124 с.

2. Горелик В.А. Анализ конфликтных ситуаций в системах управления / В.А. Горелик, М.А. Горелов, А.Ф. Кононенко. Москва. 1991.

3. Basar, T. Dynamic Noncooperative Game Theory / T. Basar, G. J. Olsder.- Philadelphia: SIAM, 1999.

4. Угольницкий Г.А. Управление устойчивым развитием активных систем / Г.А. Угольницкий. Ростов-на-Дону. 2016. 940 c.

5. Dockner E. Differential games in economics and management Science / E. Dockner, S. Jorgensen, N.V. Long, G. Sorger // Cambridge: Cambridge University Press, 2000.

6. Wright T.P. Factors affecting the cost of airplanes // Journal of the aeronautical sciences. 1936. V. 3. No. 4. P. 122128.

7. Badiru A. Computational survey of univariate and multivariate learning curve models // IEEE Transactions on Engineering Management. 1992. V. 39. No. 2. P. 176-188.

8. Yelle L.E. The learning curve: Historical review and comprehensive survey // Decision Sciences. 1979. V. 10. No. 2. P. 302-328.

9. Learning Curves: Theory, Models, and Applications / edited by Mohamad Y. Jaber. Boca Raton: CRC Press, 2011.476 $\mathrm{P}$.

10. Беллман Р. Динамическое программирование. Москва.1960.

11. Калихман И.Л. Динамическое программирование в примерах и задачах / И.Л. Калихман, М.А. Войтенко. Москва.1979. 